\title{
Galacto-Oligosaccharides Improve Barrier Function and Relieve Colonic Inflammation via Modulating Mucosa-Associated Microbiota Composition in Lipopolysaccharides-Challenged Piglets
}

\section{Ren Gao}

Nanjing Agricultural University

Shiyi Tian

Nanjing Agricultural University - Weigang Campus: Nanjing Agricultural University

Jing Wang ( $\nabla$ jwang8@njau.edu.cn )

Nanjing Agricultural University https://orcid.org/0000-0001-9496-6276

\section{Weiyun Zhu}

Nanjing Agricultural University

\section{Research}

Keywords: Barrier function, Galacto-oligosaccharides, Intestinal microbiota Lipopolysaccharides, Suckling piglets

Posted Date: February 23rd, 2021

DOl: https://doi.org/10.21203/rs.3.rs-225109/v1

License: (c) (1) This work is licensed under a Creative Commons Attribution 4.0 International License. Read Full License

Version of Record: A version of this preprint was published at Journal of Animal Science and Biotechnology on August 11th, 2021. See the published version at https://doi.org/10.1186/s40104-02100612-z. 


\section{Abstract}

\section{Background}

Galacto-oligosaccharides (GOS) have been shown to modulate the intestinal microbiota of suckling piglets to exert beneficial effects on intestinal function. However, the modulation of intestinal microbiota and intestinal function by GOS in intestinal inflammation injury models has rarely been reported. In this study, we investigated the effects of GOS on the colonic mucosal microbiota composition, barrier function and inflammatory response of lipopolysaccharides-challenged suckling piglets.

\section{Result}

we found that the colonic mucosa of LPS-challenged piglets was significantly injured and shedding, while the colonic mucosa of the LPS-GOS group piglets maintained its structure. GOS increased the relative abundance of Lactobacillus $(P=0.058)$, norank_ $f \_$Muribaculaceae and Romboutsia, and decreased the relative abundance of Alloprevotella, Campylobacter and Helicobacter in the colonic mucosa of LPS-challenged suckling piglets. In addition, GOS increased the concentrations of acetate, butyrate and total SCFAs in the colonic digesta of LPS-challenged suckling piglets. Increased SCFAs had beneficial effects on colonic barrier function and inflammatory status, including the enhanced protein expression of Claudin-1 and ZO-1 in the colonic mucosa of LPS-challenged suckling piglets, the decreased concentrations of interleukin $1 \beta$ (IL-1 $\beta$ ), interleukin 6 (IL-6), tumor necrosis factor-a (TNF- $\alpha$ ) and cluster of differentiation 14 (CD14), and the decreased relative expression of Toll-like receptor 4 (TLR4) and myeloid differentiation primary response 88 (MyD88).

\section{Conclusions}

These results suggested that GOS modulated the colonic mucosa-associated microbiota composition and improved the intestinal function of LPS-challenged suckling piglets.

\section{Introduction}

The intestinal microbiota of animals plays a key role in regulating host digestion, absorption, intestinal barrier function, and the maturation of the immune system ${ }^{1}$. Studies have shown that the intestinal microbiota of animals is established early in life, and the changes in the intestinal flora early in life can have permanent metabolic consequences for the host. Additionally, the establishment of early-life intestinal microbiota is susceptible to nutritional conditions. ${ }^{2,3}$. It suggested that the early nutrition is a primitive "driving force", which regulates the colonization of the intestinal microbiota and the development of the intestinal immune system. In the pig industry, intestinal epithelial cells of young animals are vulnerable to inflammation and infection, which negatively affects the health of animals ${ }^{4}$. Therefore, obtaining better intestinal microbial community status through nutritional intervention in early life is of great significance for the subsequent growth and health of animals. 
Galacto-oligosaccharides (GOS) are a type of functional oligosaccharide with natural properties presented abundantly in human breast milk ${ }^{5}$. As a common prebiotic, GOS are not digested and hydrolyzed in the small intestine because of their structural characteristics, but enter the hindgut, thereby are fermented and utilized by beneficial microorganisms to promote the proliferation of beneficial bacteria and contribute to the stability of intestinal microbiota ${ }^{6}$. Therefore, GOS have received more attention in recent years. According to Bouwhuis M. A. et al., dietary GOS supplementation reduced the gene expression levels of interleukin 22 (IL-22), interleukin 6खIL-6囚, and TNF- $a$ in colonic tissues of pigs challenged by Salmonella Typhimurium, which contributed to alleviating colonic inflammation in pigs ${ }^{7}$. Our previous studies have shown that the early GOS intervention can modulate colonic microbiota in suckling piglets and improve biological functions ${ }^{8}$. Therefore, whether GOS could alleviate intestinal injury in intestinal inflammation injury model is worthy of further exploration.

Lipopolysaccharides (LPS) are composed of lipids and polysaccharides in the outermost layer of the cell wall of Gram-negative bacteria and considered as a strong inflammatory stimulant ${ }^{9}$. LPS activate Nuclear factor kappa B (NF-KB) by inducing a signaling cascade that leads to the secrete of a series of inflammatory factors, including tumor necrosis factor-a (TNF-a) and IL-6, and ultimately cause the body's inflammatory response ${ }^{10}$. LPS challenge induced intestinal inflammation is a common animal inflammatory injury model ${ }^{11}$. Here, we hypothesized that GOS could modulated the intestinal microbiota of suckling piglets, alleviate inflammatory damage and maintain intestinal barrier function. In order to verify this hypothesis, we used LPS challenge suckling piglets to construct an intestinal inflammation model, and investigated the effects of GOS on colonic microbiota, inflammatory response and colonic barrier function in LPS challenged suckling piglets.

\section{Materials And Methods}

\section{Experimental design}

The experimental protocol was approved by the Animal Care and Use Committee of Nanjing Agricultural University, and all animal Care Procedures were performed in accordance with Chinese Guidelines for Animal Welfare (authorization number SYXK (Su) 2011-0036). In this study, 18 Duroc $\times$ Landrace $\times$ Large White newborn suckling piglets were selected with an average initial weight of $1.57 \pm 0.04 \mathrm{~kg}$, and they were divided into three treatment groups, CON group, LPS-CON group, and GOS-LPS group. All piglets were with sow and were free to obtain sow milk and drinking water during the experiment. Starting from the first day after birth, piglets in the GOS-LPS group were orally gavaged with $1 \mathrm{~g} / \mathrm{kg}$ body weight (BW) of GOS in the form of solution daily, and the CON group and the LPS-CON group were gavaged with equal amounts of sterile saline. The solution was carefully injected into the mouth of the piglets with a sterile medical plastic syringe. After continuous gavage for 13 days, the piglets in the LPS-CON group and the GOS-LPS group were intraperitoneally injected with $80 \mu \mathrm{g} / \mathrm{kg}$ body weight of LPS solution on the $14^{\text {th }}$ day, and the piglets in the CON group were injected with an equal volume of sterile saline. $90 \%$ pure foodgrade GOS was used in this experiment, which was purchased from Quantum Hi-Tech Biological Co., Ltd. 
(Guangdong province, China). Lipopolysaccharides (LPS, from E.Coli 055:B5) were purchased from Sigma-Aldrich (St Louis, USA).

\section{Sample collection}

Two hours after intraperitoneal injection of LPS solution or sterile saline on the $14^{\text {th }}$ day, piglets were euthanized by injection of excessive pentobarbital. The colon was separated after dissection, the colonic digesta was collected and placed in a sterile cryopreservation tube and stored in liquid nitrogen at $-80^{\circ} \mathrm{C}$ until analysis. Then the colon tissue was rinsed with cold sterile phosphate buffer saline (PBS). After PBS cleaning, the colonic mucosa was scraped with sterile slides and stored in liquid nitrogen at $-80^{\circ} \mathrm{C}$ until analysis.

\section{Histomorphological analysis of colonic epithelium}

The mid-colonic tissue samples were fixed with $4 \%$ paraformaldehyde solution and then processed with paraffin embedding technique. The Paraffin-embedded samples were cut into approximately $6 \mu \mathrm{m}$ thick sections and finally stained with hematoxylin and eosin to obtain colonic morphology ${ }^{12}$.

\section{S rRNA analysis of colonic Mucosa-Associated Microbiota}

$0.3 \mathrm{~g}$ colonic mucosa was used to extract the total bacterial DNA, and PowerSoil DNA Isolation kit (MoBio Laboratories, San Diego, U.S.) was used to isolate total DNA according to its operating instructions. The concentration and quality of DNA were determined using Nano-Drop 1000 spectrophotometer (Thermo Fisher Scientific, Waltham, Massachusetts, USA), and the extracted DNA was stored at $-80^{\circ} \mathrm{C}$ until the next analysis. The forward primer 338F (ACTCCTACGGGAGGCAGCAG) and reverse primer 806R (GGACTACHVGGGTWTCTAAT) were used to amplify the V3-V4 region of the bacterial 16S rRNA gene. DNA was amplified using ABI GeneAmp 9700 PCR Thermocycle Instrument (Applied Biosystems, Inc., Carlsbad, USA) with specific reaction parameters as follows: first pre-deformation stage (3 minutes at $95^{\circ} \mathrm{C}$ ), then 30 seconds at $95^{\circ} \mathrm{C} ; 30$ seconds at $55^{\circ} \mathrm{C} ; 45$ seconds at $72^{\circ} \mathrm{C}$ for 27 cycles, and finally 10 minutes at $72^{\circ} \mathrm{C}$. Next, the PCR products were purified by $2 \%$ agarose gel electrophoresis, and the PCR products were cut and recovered using the AxyPrep DNA Gel Recovery Kit (Axygen, Union City, USA). According to the preliminary quantitative results of electrophoresis, the PCR products were quantified by QuantiFluor-ST Handheld Fluorometer with UV/Blue Channels (Promega, Madison, Wisconsin, USA). Finally, the PCR product was sequenced on Miseq (Illumina Inc., San Diego, USA) after concentration normalization. The $16 \mathrm{~S}$ rRNA raw reads in this study were uploaded to GenBank in NCBI and the accession number is SRP297862.

\section{Bioinformatics analysis}

The PE reads obtained by Miseq sequencing were spliced according to the overlap relationship, and the sequence quality was controlled and filtered at the same time. Operational taxonomic units (OTUs) were clustered with $97 \%$ similarity cut-off using Usearch (version 7.0.1090, http://www.drive5.com/uparse/). 
The OTU sequences were classified using RDP Classifier (version 2.11, https://sourceforge.net/projects/rdp-classifier/) with confidence threshold of $70 \%$. Alpha diversity of colonic mucosal microbiota was analyzed with Mothur (version 1.30.2, https://www.mothur.org/wiki/Download_mothur), including Shannon, Simpson, ace and Chao index. Principal coordinate analysis ( $\mathrm{PCOA}$ ) was performed based on bray-curtis distance, and then the analysis of ANOSIM was conducted based on bray-curtis distance to assess significant differences among samples by using Qiime (version 1.9.1, http://qiime.org/install/index.html).

\section{Measurement of pH value and microbial metabolites in colonic digesta}

During the sampling process, the colonic digesta was collected with sterilized centrifuge tube, and the $\mathrm{pH}$ value was determined immediately with a pH meter (PB-10, Sartorius Group, Gottingen, Germany). According to the description of Shi et al, a gas chromatography method was used to determine the concentration of acetate, propionate, isobutyrate, butyrate, isovalerate, valerate and total short chain fatty acids in colonic digesta ${ }^{13}$. The concentration of lactic acid in colonic digesta was determined by using the reagent kit (Nanjing Jiancheng Technology Co., Ltd., Nanjing, China) according to its operating instructions.

\section{Quantitative real-time PCR}

The total RNA was extracted from $0.2 \mathrm{~g}$ colonic mucosa with TRIzol reagent (Takara Bio, Japan) according to the operating instructions. A Nano-Drop 1000 spectrophotometer (Thermo Fisher Scientific, Waltham, Massachusetts, USA) was used to determine the concentration and quality of RNA. After concentration normalization, RNA was reversely transcribed into cDNA using HiScript * III RT SuperMix for qPCR reagent kit (Vazyme Biotech, Nanjing, China) and stored at $-80^{\circ} \mathrm{C}$. The target genes were detected by fluorescence quantitative PCR using ChamQ SYBR qPCR Master Mix reagent kit (Vazyme Biotech, Nanjing, China) on ABI StepOnePlus real-time fluorescence quantitative PCR instrument (Applied Biosystems, Inc., Carlsbad, USA). Primers for this experiment were synthesized in Invitrogen Life Technologies (Shanghai, China) and their sequences are shown in Table 1. Finally, the relative expression of the target gene was calculated by the $2^{-\Delta \Delta C t}$ method ${ }^{14}$. Glyceraldehyde-3-phosphate dehydrogenase (GAPDH) was used as the housekeeping gene.

\section{Measurement of mucosal cytokine and cluster of differentiation 14 (CD14)}

$0.1 \mathrm{~g}$ colonic mucosa tissue with $1000 \mu \mathrm{L}$ of pre-cooled PBS was homogenized to extract total protein. The crushed tissue was centrifuged at $10,000 \mathrm{rpm}$ for $15 \mathrm{~min}$ at $4^{\circ} \mathrm{C}$, and then the supernatant was collected for further analysis. The protein concentration was determined by BCA method with the protein detection kit (Biosharp life science, Hefei, China). Finally, the concentration of interleukin $1 \beta$ (IL-1 $\beta$ ), interleukin 6 (IL-6), interleukin 8 (IL-8), interleukin 10 (IL-10), tumor necrosis factor-a (TNF-a), transforming growth factor- $\beta$ (TGF- $\beta$ ) and CD14 in the colonic mucosa was determined using the Enzyme Linked Immunosorbent Assay (ELISA) kit (R\&D Systems Inc., MN, USA) according to its operating instructions. 


\section{Western blot analysis}

$0.1 \mathrm{~g}$ of colonic mucosal tissue in $500 \mu \mathrm{L}$ RIPA lysis buffer with $1 \%$ protease inhibitors (FUTURE SCIENTIFIC INNOVATION, Nanjing, China) was homogenized at $4^{\circ} \mathrm{C}$. The pulverized tissue was centrifuged at $12000 \mathrm{~g}$ for $15 \mathrm{~min}$ at $4^{\circ} \mathrm{C}$, and the supernatant was collected for Western blot analysis. For normalization, the protein concentration was determined with the BCA protein assay kit (Biosharp life science, Hefei, China). After normalization, $5 \times$ loading buffer in a ratio of 1:4 was added and the proteins were boiled for 5 minutes to denature. The proteins were separated by $12 \%$ SDS-PAGE after cooling, and then electrotransferred onto polyvinylidene fluoride (PVDF) membranes (Merck Millipore, Darmstadt, Germany). After the transfer, the membrane was blocked with 1×Tris-buffered saline-Tween (TBST) buffer containing $5 \%$ fat-free milk at room temperature for 1 hour, and then the membrane was incubated with the primary antibody overnight at $4^{\circ} \mathrm{C}$. The specific primary antibodies used included beta-actin ( $\beta$-actin) (Cell Signaling Technology, Danvers, USA), Zona occludens protein 1 (ZO-1), Occludin, and Claudin1(Proteintech, Chicago, USA). After overnight, the membrane was washed three times with $1 \times$ TBST for 10 minutes each, and then incubated with antirabbit IgG HRP-conjugated secondary antibody (1:1000; Cell Signaling Technology, Danvers, U.S.) at room temperature for 1 hour. After secondary antibody incubation, the membrane was washed three times with TBST for 10 minutes each time. Finally, the target bands were visualized through an Automatic chemiluminescence/fluorescence image analysis system (Tanon 5200 Multi, Shanghai, China). The gray value of band was measured by the ImageJ (version 1.8.0), and the relative abundance of each target protein was expressed as the target protein/ $\beta$ actin protein ratio.

\section{Statistical analysis}

All data were subjected to one-way analysis of variance for a completely randomized design using the general linear model procedure of SPSS (version 20, IBM, Chicago, USA) and expressed as the means \pm standard error of mean (SEM). Statistical differences among the treatments were separated by Tukey test. Statistical significance was set at $P<0.05$, whereas $P$ values between 0.05 and 0.10 were considered as a trend.

\section{Results}

\section{Colonic morphology}

As shown in Figure 1, compared with the CON group, LPS challenge resulted in an obvious damage and shedding of colonic mucosa (Figure 1b). However, the colonic mucosa of piglets in the LPS-GOS group was only slightly injured and shed (Figure 1c). There were no significant differences in colonic crypt depth and mucosal thickness among the three groups (Table 2).

\section{Diversity of the colonic mucosa-associated microbiota}


In this study, we obtained a total of 713,963 sequences in the V3-V4 region, with an average of 39665 sequences per sample. The OTU was clustered with $97 \%$ similarity. There were 768,685 and 791 core OTUs in the CON group, LPS-CON group and LPS-GOS group, respectively, and 465 core OTUs were common in the three groups (Figure 2a). The effects of three treatments on colonic mucosa-associated microbiota alpha and beta diversity were investigated. As shown in Figure 2c-2f, the a diversity index including Shannon, Simpson, Ace and Chao indexes were not significantly different among the three groups. $\beta$ diversity, PCoA analysis based on bray-curtis distance, showed significant separations of colonic mucosal microbial composition among the three groups (Figure $2 b$ ).

\section{Bacterial abundance in the colonic mucosa}

The colonic mucosa microbiota composition in the three treatment groups is shown in Figure 3. At the phylum level, Firmicutes, Bacteroidetes, and Proteobacteria are the mainly dominant phyla (Figure 3a). Statistical analysis showed that the relative abundance of Firmicutes in the LPS-CON group tended to decrease $(P=0.054)$ while the relative abundance of Actinobacteria increased significantly $(P<0.05)$ compared with the CON group. However, the relative abundance of Firmicutes in the LPS-GOS group tended to increase $(P=0.059)$, and the relative abundance of Actinobacteria tended to decrease (Figure $3 \mathrm{~b}, 3 \mathrm{c})(P=0.086)$ compared with the LPS-CON group.

At the genus level, genera with relative abundance greater than $0.1 \%$ in at least one treatment group are shown in Figure 4a. According to the statistical analysis, LPS challenge significantly reduced the relative abundance of norank_f_Muribaculaceae, Prevotellaceae_NK3B31_group, and Ruminococcaceae_NK4A214_group $(P<0.05)$, tended to reduce the relative abundance of Lactobacillus $(P=0.097)$ and Romboutsia $(P=0.083)$ (Figure $4 \mathrm{~b}-4 \mathrm{f})$ compared with the CON group. In addition, the relative abundance of Alloprevotella, Campylobacter and Helicobacter $(P<0.01)$ significantly increased and the relative abundance of Alistipes $(P=0.085)$ tended to increase (Figure $4 \mathrm{~g}-4 \mathrm{j})$ in the LPS-CON group. On the other hand, the relative abundance of norank_ $f$ _Muribaculaceae $(P<0.01)$ and Romboutsia $(P<0.05)$ in the LPS-GOS group increased significantly, and the relative abundance of Lactobacillus ( $P=0.058$ ) tended to increase (Figure 4b, 4c, 4f) compared with the LPS-CON group. In addition, the relative abundance of Alloprevotella, Alistipes $(P<0.05)$, Campylobacter and Helicobacter $(P$ $<0.01$ ) decreased significantly (Figure 4g-4j).

\section{The pH value andmicrobial metabolites in colonic digesta}

The effect of GOS on pH value and microbial metabolites of colonic digesta in suckling piglets challenged with LPS are shown in Table 2. Compared with the CON group, LPS challenge significantly increased the $\mathrm{pH}$ value $(P<0.05)$ of colonic digesta, significantly reduced the concentrations of acetate, butyrate $(P<0.05)$ and total SCFAs $(P<0.01)$ in colonic digesta, and tended to reduce the concentration of lactate $(P=0.051)$. However, compared with the LPS-CON group, the $\mathrm{pH}$ of colonic digesta was significantly decreased $(P<0.05)$ and the concentrations of acetate, butyrate, total SCFAs and lactate in the colonic digesta were significantly increased $(P<0.05)$ in the LPS-GOS group. 


\section{The mRNA expression of mucins in the colonic mucosa}

As shown in Figure 5, the relative mRNA expression of Mucin 1 (MUC1) and Mucin 2 (MUC2) in colonic mucosa of piglets in the LPS-CON group was significantly higher than that in the CON group $(P<0.05)$. Compared with the LPS-CON group, the relative mRNA expression of $M U C 1$ in the LPS-GOS group tended to decrease $(P=0.080)$, and the relative mRNA expression of MUC2 significantly decreased $(P<0.05)$. There was no significant change in MUC4 expression among the three groups.

\section{The protein expression level of tight junction protein in colonic mucosa}

As shown in Figure 6, the relative protein expression of Claudin-1 and ZO-1 in the colonic mucosa were significantly decreased in the LPS-CON group compared with those in the CON group $(P<0.05)$. In addition, the relative protein expression of Claudin-1 and ZO-1 were significantly increased in the LPSGOS group compared with those in the LPS-CON group $(P<0.05)$. The relative protein expression of Occludin was not significantly different among the three groups.

\section{The concentration of cytokines in the colonic mucosa}

As shown in Figure 7a-7c and 7e, LPS challenge significantly increased the concentrations of IL-1 $\beta$, IL-6, IL-8 $(P<0.05)$ and TNF-a $(P<0.01)$ in colonic mucosa compared with the CON group. The concentrations of IL-1 $\beta$, IL-6 $(P<0.05)$ and TNF-a $(P<0.01)$ in the LPS-GOS group were decreased significantly, and the concentration of IL-8 $(P=0.068)$ tended to decrease compared with those in the LPS-CON group. No significant difference was observed in the concentration of IL-10 and TGF- $\beta$ among the three groups (Figure 7d, 7f).

\section{The concentration of CD14 in colonic mucosa and the relative mRNA expression of key molecules in NF- KB signaling pathway}

As shown in Figure 8a-8d, LPS challenge significantly increased the concentration of CD14 $(P<0.01)$ and the relative mRNA expression of TLR4, MyD88 and NF-KB $p 65(P<0.05)$ in the colonic mucosa compared with the CON group. The concentration of CD14 in the colonic mucosa was significantly decreased and the relative mRNA expressions of TLR4 and MyD88 in the colonic mucosa were significantly decreased in the LPS-GOS group while the relative mRNA expression of NF-KB p65 tended to decrease $(P=0.062)$ compared with those in the LPS-CON group.

\section{Discussion}

The first week after birth is a critical period for the colonization of intestinal microbiota in piglets, and the colonization and succession of the intestinal microbiota in early life is the key factor that affects the establishment of specific microbiota composition and phenotype of newborns ${ }^{15}, 16$. In addition, the development of innate and adaptive immune responses, as well as the homeostasis of intestinal barrier function are largely influenced by intestinal microbial colonization ${ }^{17}$. As we know, the colon is the 
intestinal segment with the largest number of microorganisms and vigorous microbial metabolic activity 18. It has been pointed out that the intestinal microbiota has the most significant regulatory effect on intestinal immunity of piglets in the colon ${ }^{19}$. Intraperitoneal injection of LPS is widely used to construct an animal model of acute intestinal inflammatory injury ${ }^{11}$. Thus, we have investigated the beneficial effects of GOS in piglet colonic inflammation injury model with LPS challenge. In this study, we found that LPS challenge caused colonic epithelial tissue injury by morphological analysis, while GOS alleviated colonic tissue injury. In order to understand the relationship between the intestinal microbiota with intestinal immunity and barrier function, we focused on investigating the effect of GOS on the colonization of colonic microbiota, colonic inflammatory response and barrier function in LPS-challenged suckling piglets.

In the present study, we observed that Firmicutes and Bacteroidetes were the most abundant phyla in the colonic mucosa of suckling piglets, which was consistent with the study of Li et al ${ }^{20}$. The relative abundance of Firmicutes was decreased and Actinobacteria was increased in the LPS-CON group compared with that in the CON group. However, the relative abundance of Firmicutes was increased and Actinobacteria was decreased in the LPS-GOS group compared with that in the LPS-CON group. Increased relative abundance of Firmicutes is beneficial to intestinal health, as it contains a large number of SCFAproducing bacteria, such as butyrate-producer Eubacterium rectale and Eubacterium Hallii, which help to maintain intestinal health ${ }^{21}$. Actinobacteria is reported to be part of the symbiosis intestinal microbiota, but a high proportion of Actinobacteria is associated with intestinal inflammatory diseases and colon cancer ${ }^{22}$. Therefore, our results suggested that GOS could increased the relative abundance of Firmicutes and decreased the relative abundance of Actinobacteria in the colonic mucosa of LPSchallenged piglets.

At the genus level, we found that LPS stimulation resulted in the changes of the relative abundance of nine genera, and the changes in seven genera were reversed by GOS intervention. LPS challenge decreased the relative abundance of Lactobacillus and norank_f__Muribulaceae and increased the relative abundance of Alloprevotella, while GOS intervention prevented the LPS-induced reduction of the relative abundance of Lactobacillus and norank_f_Muribulaceae and the increase of Alloprevotella. Lactobacillus is a common probiotic that has been shown to colonize the intestine and become a stable member of the entire intestinal microbial community shortly after birth in piglets ${ }^{23}$. Miyauchi et al. have reported that Lactobacillus could maintain the colonic epithelial barrier function and alleviate the colonic inflammatory response in colitis mice ${ }^{24}$. In addition, Lactobacillus would prevent intestinal pathogen infection and protect intestinal health by producing metabolites such as lactic acid and competing for mucosal binding sites with Gram-negative pathogens such as Campylobacter ${ }^{25}$, 26 . Our results suggest that the increased relative abundance of Lactobacillus may contribute to the protective effect of GOS on colon health in LPS-challenged suckling piglets. The function of norank_f_Muribaculaceae is largely unknown, but Liu et al. found that the relative abundance of norank_f_Muribaculaceae was positively correlated with the metabolites enriched in the feces of healthy mice ${ }^{27}$. This suggested that norank_f_Muribaculaceae might be a bacterium enriched in the healthy intestinal environment. The 
relative abundance of Alloprevotella increased in the colon mucosa of ulcerative colitis carcinogenesis mice ${ }^{28}$. Additionally, our results are consistent with Wang's finding that the early GOS intervention can reduce the relative abundance of Alloprevotella in the colonic mucosa of suckling piglets ${ }^{8}$. Hence, we speculated that the significant decrease in the relative abundance of Alloprevotella in the LPS-GOS group compared with that in the LPS-CON group might be an indication of GOS alleviating the colonic inflammation caused by LPS in piglets. Notably, LPS stimulation led to an increase in the relative abundance of potential pathogenic bacteria Alistipes, Campylobacter and Helicobacter, but GOS could significantly reduce their relative abundance. Alistipes, Campylobacter and Helicobactercan cause inflammatory diseases in the intestine, resulting in gastrointestinal dysfunction ${ }^{29-31}$. Therefore, in general, our results suggested that GOS contributed to maintaining colonic mucosal microbiota homeostasis in LPS-challenged suckling piglets, promoting the colonization of beneficial bacteria and reducing the colonization of potentially pathogenic bacteria.

SCFAs are main metabolites of intestinal microorganisms. Through the production of metabolites including SCFAs, lactic acid and bile acid, the intestinal microbiota established a close association with various physiological and immune responses of the host ${ }^{32}$. Here, we observed significant changes in the concentrations of acetate, butyrate and total SCFAs in the colonic lumen. LPS significantly reduced the concentrations of acetate, butyrate, and total SCFAs. This may be due to the fact that LPS reduced the relative abundance of Firmicutes, Romboutsia, Prevotellaceae_NK3B31_group and Ruminococcaceae_NK4A214_group, which produce SCFAs including acetate and butyrate by fermenting dietary components ${ }^{21,33-35}$. GOS increased the concentration of SCFAs by significantly increasing the relative abundance of Firmicutes and Romboutsia in LPS-challenged suckling piglets. SCFAs are key factors regulating the intestinal epithelial barrier function and intestinal immunity of the host, and have a variety of beneficial effects on the host, including maintaining the intestinal barrier function and antiinflammatory effects ${ }^{36}$. Meanwhile, the stability of colonic mucosa is largely influenced by the concentration of SFCAs which are produced by colonic microbiota. Butyrate, which is a major nutrient involved in the repair and regeneration of colonic epithelial cells, plays an important role in the health of colonic mucosa ${ }^{37,38}$. In addition, we found that LPS decreased the concentration of lactate in the colonic lumen, while GOS significantly increased the concentration of lactate, which was consistent with the change trend of Lactobacillus. Neal-McKinney et al. reported that lactate is an important factor for reducing Campylobacter growth in livestock ${ }^{39}$. Our finding of the decreased relative abundance of Campylobacter in the colonic mucosa was consistent with this idea. Notably, lactate can be further metabolized to SCFAs, especially butyrate, by lactate-utilizing bacteria such as Megasphaera in the intestine ${ }^{40,41}$. With the increase of SCFAs and lactate concentration, the pH value of colon in the LPSGOS group decreased significantly compared with that in the LPS-CON group. A lower $\mathrm{pH}$ value is beneficial to maintain the acidic environment in the colon, thereby inhibiting the growth and colonization of pathogenic bacteria such as E. coli and Salmonella ${ }^{42}$. Therefore, the increased concentrations of SCFAs and lactate in the LPS-GOS group piglets contributed to the colonic mucosal homeostasis. 
SCFAs have been shown to exert effective anti-inflammatory effects in the intestine ${ }^{43}$. In order to further explore the inflammatory status of the piglet colon, we determined the levels of cytokines. We found that LPS stimulation significantly increased the concentrations of pro-inflammatory cytokines IL-1 $\beta$, IL-6, IL-8 and TNF- $a$ in the colonic mucosa, without significant effect on the anti-inflammatory cytokines IL-10 and TGF- $\beta$, which indicated that LPS promoted the secretion of proinflammatory cytokines to induce inflammatory response. However, GOS significantly inhibited the increase of IL-1 $1 \beta$, IL-6, IL-8 and TNF-a concentration caused by LPS. Studies have shown that LPS induce the release of a series of proinflammatory cytokines through binding to TLR4 and stimulating the downstream signaling molecule MyD88 as well as NF-KB p65 ${ }^{44}$. Zanoni et al. found that activation of TLR4/NF-KB pathway caused by bacterial endotoxin in the traditional sense was regulated by CD14 because of the high affinity between CD14 and LPS. CD14 is the first pattern recognition receptor that binds to LPS, can transmit LPS to TLR4 and control TLR4 entry into the endosomal network through endocytosis ${ }^{45}$. In this study, we found that the decreased concentrations of pro-inflammatory cytokines IL-1 $\beta$, IL-6, IL-8 and TNF- $\alpha$ in the LPS-GOS group were consistent with the decreased concentration of CD14 and the relative mRNA expression of $T L R 4, M y D 88$ and NF-KB $p 65$ in the colonic mucosa. The NF-KB signaling pathway plays a key regulatory role in the inflammatory response, and Meijer et al. have found that SCFAs can inhibit the activation of NF-KB and contribute to the reduced secretion of pro-inflammatory cytokines ${ }^{46}$. Thus, in our study, GOS supplement increased the relative abundance of SCFAs producing bacteria, accompanied by an increase in a higher concentration of SCFAs, which may contribute to the reduced secretion of pro-inflammatory cytokines in LPS-challenged suckling piglets.

Altered intestinal microbiota and SCFAs have important effects on the maintenance of intestinal barrier function ${ }^{47}$. In addition, intestinal inflammation is closely associated with the intestinal barrier. IL-1 $\beta$, IL- 6 and IL-8 were reported to impair intestinal barrier function by rearranging tight junction proteins, while TNF-a significantly disrupts the tight junctions between intestinal epithelial cells ${ }^{48}$. Hence, GOS alleviated the colonic inflammation caused by LPS challenge in piglets, which contributed to the maintenance of colonic mucosal barrier function. Intestinal tight junctions (TJs) mainly include Claudin, Occludin and zonula occludens (ZO) families, which are the main components of the physical barrier of intestinal epithelium and determine the selective permeability of intestinal epithelium ${ }^{49}$. TJs are vulnerable to the exposure of external stressors, which cause unregulated transport and diffusion of macromolecules such as endotoxin and antigen into mucosa, leading to local or systemic inflammation ${ }^{50}$. This explains why LPS stimulation significantly reduced the protein expression of Claudin-1 and ZO-1 in present study. Some studies have reported that probiotics such as Lactobacillus plantarum can increase the expression of TJs in intestinal epithelium of piglets, and SCFAs could enhance intestinal barrier function by activating AMPK signaling pathway ${ }^{51,52}$. In our study, the protein expression of Claudin-1 and ZO-1 in the LPS-GOS group was significantly higher than that in the LPS-CON group. Mucins play a key role in the chemical barrier formed by the epithelial mucus layer, and the number and maturity of mucins covering the intestinal epithelial surface are important factors influencing the optimal disease resistance ${ }^{53}$. Interestingly, we found that the relative mRNA expression of $M U C 1$ and $M U C 2$ in the LPS-CON group was 
significantly higher than that in the CON group. Studies have pointed out that LPS stimulation can cause mucosal mucin secretion to be excessive, and this change is usually accompanied by bacterial inflammation of the mucosa ${ }^{54}$. Therefore, we speculated that the significant increase of the relative mRNA expression of MUC1 and MUC2 were associated with LPS which stimulate piglets to produce an acute inflammation response 2 hours after intraperitoneal injection, and resulted in a large amount of mucus secreted by the colon in this study. The relative mRNA expression of MUC1 and MUC2 in the LPSGOS group was decreased compared with that in the LPS-CON group, probably because GOS help to alleviate the inflammation response caused by LPS. Therefore, GOS help to maintain the colonic epithelial barrier function in LPS-challenged suckling piglets, which may be mainly related to the changes of colonic mucosa-associated microbiota composition and metabolites, as well as the alleviation of inflammation.

\section{Conclusion}

In summary, this study demonstrated that GOS supplement modulated the colonic mucosal microbiota in LPS-challenged suckling piglets, which was beneficial to the construction of a healthy colonic environment. In addition, GOS increased the concentration of SCFAs in the colonic lumen, and contributed to alleviate LPS-induced colonic inflammatory response and ultimately help to maintain the colonic barrier function.

\section{Abbreviations}

GOS, galacto-oligosaccharides; LPS, lipopolysaccharides; MUC1, Mucin 1; MUC2, Mucin 2; MUC4, Mucin 4; TLR4, Toll-like receptor 4; Myd88, myeloid differentiation primary response 88; NF-kB-p65, nuclear factor kappa B-p65; GAPDH, glyceraldehyde phosphate dehydrogenase; TJs, tight junctions; ZO-1, zonula occluden 1 ; CD14, cluster of differentiation 14; IL-1 $\beta$, interleukin $1 \beta$; IL-6, interleukin 6; IL-8, interleukin 8; IL10 , interleukin 10; TNF- $a$, tumor necrosis factor- $\alpha$; TGF- $\beta$, transforming growth factor- $\beta$.

\section{Declarations}

\section{Acknowledgements}

The authors thank the National Center for International Research on Animal Gut Nutrition for financial support.

\section{Corresponding Author}

*Tel.: +86-25-84395523. Fax: +86-25-84395314. Email: jwang8@njau.edu.cn

\section{Funding}

This study was supported by the National Key R\&D Program of China 2017YFD0500505. 
Availability of data and materials

All data generated or analyzed during this study are available from the corresponding author upon reasonable request.

\section{Authors' contributions}

R G and SY T led the design and performance of the experiments, the analysis of the data, the writing of the paper, and the measurement of indicators. J W and WY Z participated in designing the experiments and editing the paper. All authors read and approved the final manuscript.

\section{Competing interests}

The authors declare that they have no competing interests.

\section{Consent for publication}

Not applicable.

\section{References}

(1) L. F. Stinson, Establishment of the early-life microbiome: a DOHaD perspective, J Dev Orig Hlth Dis, 2019, 11, 201-210.

(2) L. M. Cox, S. Yamanishi, J. Sohn, A. V. Alekseyenko, J. M. Leung, I. Cho, S. G. Kim, H. Li, Z. Gao, D. Mahana, J. G. Z. Rodriguez, A. B. Rogers, N. Robine, P. Loke and M. J. Blaser, Altering the Intestinal Microbiota during a Critical Developmental Window Has Lasting Metabolic Consequences, Cell, 2014, $158,705-721$.

(3) D. Schokker, J. Zhang, L. L. Zhang, S. A. Vastenhouw, H. G. H. J. Heilig, H. Smidt, J. M. J. Rebel and M. A. Smits, Early-Life Environmental Variation Affects Intestinal Microbiota and Immune Development in New-Born Piglets, Plos One, 2014, 9.

(4) P. T. Sangild, Development of the mammalian gastrointestinal tract, the resident microbiota, and the role of diet in early life, European Journal of Cancer Supplements, 2011, 3, 85.

(5) A. M. Zivkovic and D. Barile, Bovine Milk as a Source of Functional Oligosaccharides for Improving Human Health, Adv Nutr, 2011, 2, 284-289.

(6) D. P. M. Torres, M. P. Goncalves, J. A. Teixeira and L. R. Rodrigues, Galacto-Oligosaccharides:

Production, Properties, Applications, and Significance as Prebiotics, Compr Rev Food Sci F, 2010, 9, 438454.

(7) M. A. Bouwhuis, M. J. McDonnell, T. Sweeney, A. Mukhopadhya, C. J. O'Shea and J. V. O'Doherty, Seaweed extracts and galacto-oligosaccharides improve intestinal health in pigs following Salmonella 
Typhimurium challenge, Animal, 2017, 11, 1488-1496.

(8) J. Wang, S. Y. Tian, H. Yu, J. Wang and W. Y. Zhu, Response of Colonic Mucosa-Associated Microbiota Composition, Mucosal Immune Homeostasis, and Barrier Function to Early Life Galactooligosaccharides Intervention in Suckling Piglets, J Agr Food Chem, 2019, 67, 578-588.

(9) M. Zhang, H. C. Pan, Y. J. Xu, X. T. Wang, Z. H. Qiu and L. Jiang, Allicin Decreases LipopolysaccharideInduced Oxidative Stress and Inflammation in Human Umbilical Vein Endothelial Cells through Suppression of Mitochondrial Dysfunction and Activation of Nrf2, Cell Physiol Biochem, 2017, 41, 22552267.

(10) Y. C. Lu, W. C. Yeh and P. S. Ohashi, LPS/TLR4 signal transduction pathway, Cytokine, 2008, 42, 145151.

(11) X. Y. Wang, W. J. Wang, L. M. Wang, C. Yu, G. L. Zhang, H. L. Zhu, C. W. Wang, S. J. Zhao, C. A. A. Hu and Y. L. Liu, Lentinan modulates intestinal microbiota and enhances barrier integrity in a piglet model challenged with lipopolysaccharide, Food Funct, 2019, 10, 479-489.

(12) D. Y. Wang, S. Y. Xu, Y. Lin, Z. F. Fang, L. Q. Che, B. Xue and D. Wu, Recombinant porcine epidermal growth factor-secreting Lactococcus lactis promotes the growth performance of early-weaned piglets, Bmc Vet Res, 2014, 10.

(13) C. Shi, Y. Zhu, Q. Niu, J. Wang, J. Wang and W. Zhu, The Changes of Colonic Bacterial Composition and Bacterial Metabolism Induced by an Early Food Introduction in a Neonatal Porcine Model, Current Microbiology, 2018, 75, 745-751.

(14) T. D. Schmittgen and K. J. Livak, Analyzing real-time PCR data by the comparative C-T method, Nat Protoc, 2008, 3, 1101-1108.

(15) T. Gensollen, S. S. lyer, D. L. Kasper and R. S. Blumberg, How colonization by microbiota in early life shapes the immune system, Science, 2016, 352, 539-544.

(16) N. Mach, M. Berri, J. Estelle, F. Levenez, G. Lemonnier, C. Denis, J. J. Leplat, C. Chevaleyre, Y. Billon, J. Dore, C. Rogel-Gaillard and P. Lepage, Early-life establishment of the swine gut microbiome and impact on host phenotypes, Env Microbiol Rep, 2015, 7, 554-569.

(17) L. V. Hooper and A. J. Macpherson, Immune adaptations that maintain homeostasis with the intestinal microbiota, Nat Rev Immunol, 2010, 10, 159-169.

(18) A. M. O'Hara and F. Shanahan, The gut flora as a forgotten organ, Embo Rep, 2006, 7, 688-693.

(19) S. H. Duncan, G. E. Lobley, G. Holtrop, J. Ince, A. M. Johnstone, P. Louis and H. J. Flint, Human colonic microbiota associated with diet, obesity and weight loss, Int J Obesity, 2008, 32, 1720-1724. 
(20) N. Li, S. M. Huang, L. L. Jiang, W. Wang, T. T. Li, B. Zuo, Z. Li and J. J. Wang, Differences in the Gut Microbiota Establishment and Metabolome Characteristics Between Low- and Normal-Birth-Weight Piglets During Early-Life, Front Microbiol, 2018, 9.

(21) P. Louis and H. J. Flint, Formation of propionate and butyrate by the human colonic microbiota, Environ Microbiol, 2017, 19, 29-41.

(22) Q. Zhang, J. Li, M. Cao, Y. Li, Y. Zhuo, Z. Fang, L. Che, S. Xu, B. Feng, Y. Lin, X. Jiang, X. Zhao and Wu, Dietary supplementation of Bacillus subtilis PB6 improves sow reproductive performance and reduces piglet birth intervals, Anim Nutr, 2020, 6, 278-287.

(23) R. Kenworthy and W. E. Crabb, The Intestinal Flora of Young Pigs, with Reference To Early Weaning, Escherichia Coli and Scours, J Comp Pathol, 1963, 73, 215-228.

(24) E. Miyauchi, H. Morita and S. Tanabe, Lactobacillus rhamnosus alleviates intestinal barrier dysfunction in part by increasing expression of zonula occludens- 1 and myosin light-chain kinase in vivo, J Dairy Sci, 2009, 92, 2400-2408.

(25) E. Mann, S. Schmitz-Esser, Q. Zebeli, M. Wagner, M. Ritzmann and B. U. Metzler-Zebeli, Mucosaassociated bacterial microbiome of the gastrointestinal tract of weaned pigs and dynamics linked to dietary calcium-phosphorus, Plos One, 2014, 9, e86950.

(26) A. Savadogo, C. A. T. Ouattara, I. H. N. Bassole and S. A. Alfred, Bacteriocins and lactic acid bacteria a minireview, Afr J Biotechnol, 2006, 5, 678-U671.

(27) Y. Liu, Y. K. Luo, X. H. Wang, L. Y. Luo, K. Sun and L. Zeng, Gut Microbiome and Metabolome Response of Pu-erh Tea on Metabolism Disorder Induced by Chronic Alcohol Consumption, J Agr Food Chem, 2020, 68, 6615-6627.

(28) C. Wang, W. Li, H. Wang, Y. Ma, X. Zhao, X. Zhang, H. Yang, J. Qian and J. Li, Saccharomyces boulardii alleviates ulcerative colitis carcinogenesis in mice by reducing TNF-alpha and IL-6 levels and functions and by rebalancing intestinal microbiota, BMC Microbiol, 2019, 19, 246.

(29) C. Kong, R. Gao, X. Yan, L. Huang and H. Qin, Probiotics improve gut microbiota dysbiosis in obese mice fed a high-fat or high-sucrose diet, Nutrition, 2019, 60, 175-184.

(30) W. Zhang, Y. H. Zhu, D. Zhou, Q. Wu, D. Song, J. Dicksved and J. F. Wang, Oral Administration of a Select Mixture of Bacillus Probiotics Affects the Gut Microbiota and Goblet Cell Function following Escherichia coli Challenge in Newly Weaned Pigs of Genotype MUC4 That Are Supposed To Be Enterotoxigenic E. coli F4ab/ac Receptor Negative, App/ Environ Microbiol, 2017, 83.

(31) S. Krakowka, K. A. Eaton and D. M. Rings, Occurrence of gastric ulcers in gnotobiotic piglets colonized by Helicobacter pylori, Infect Immun, 1995, 63, 2352-2355. 
(32) B. Liu, W. Wang, X. Zhu, X. Sun, J. Xiao, D. Li, Y. Cui, C. Wang and Y. Shi, Response of Gut Microbiota to Dietary Fiber and Metabolic Interaction With SCFAs in Piglets, Front Microbiol, 2018, 9, 2344.

(33) L. R. Lopetuso, F. Scaldaferri, V. Petito and A. Gasbarrini, Commensal Clostridia: leading players in the maintenance of gut homeostasis, Gut Pathog, 2013, 5, 23.

(34) X. Wei, J. Tao, S. Xiao, S. Jiang, E. Shang, Z. Zhu, D. Qian and J. Duan, Xiexin Tang improves the symptom of type 2 diabetic rats by modulation of the gut microbiota, Sci Rep, 2018, 8, 3685.

(35) C. Kang, B. Wang, K. Kaliannan, X. Wang, H. Lang, S. Hui, L. Huang, Y. Zhang, M. Zhou, M. Chen and M. Mi, Gut Microbiota Mediates the Protective Effects of Dietary Capsaicin against Chronic Low-Grade Inflammation and Associated Obesity Induced by High-Fat Diet, mBio, 2017, 8.

(36) C. L. Maynard, C. O. Elson, R. D. Hatton and C. T. Weaver, Reciprocal interactions of the intestinal microbiota and immune system, Nature, 2012, 489, 231-241.

(37) M. A. Franklin, A. G. Mathew, J. R. Vickers and R. A. Clift, Characterization of microbial populations and volatile fatty acid concentrations in the jejunum, ileum, and cecum of pigs weaned at 17 vs 24 days of age, J Anim Sci, 2002, 80, 2904-2910.

(38) M. Lopez-Siles, S. H. Duncan, L. J. Garcia-Gil and M. Martinez-Medina, Faecalibacterium prausnitzii: from microbiology to diagnostics and prognostics, ISME J, 2017, 11, 841-852.

(39) J. M. Neal-McKinney, X. Lu, T. Duong, C. L. Larson, D. R. Call, D. H. Shah and M. E. Konkel, Production of organic acids by probiotic lactobacilli can be used to reduce pathogen load in poultry, Plos One, 2012, 7, e43928.

(40) K. Ushida, K. Ushida and K. Ushida, 13 C-NMR Studies on Lactate Metabolism in a Porcine Gut Microbial Ecosystem, Microbial Ecology in Health \& Disease, 2002, 14, 242-247.

(41) T. Tsukahara, K. Hashizume, H. Koyama and K. Ushida, Stimulation of butyrate production through the metabolic interaction among lactic acid bacteria, Lactobacillus acidophilus, and lactic acid-utilizing bacteria, Megasphaera elsdenii, in porcine cecal digesta, Animal Science Journal, 2006.

(42) N. Shibata, J. Kunisawa and H. Kiyono, Dietary and Microbial Metabolites in the Regulation of Host Immunity, Front Microbiol, 2017, 8, 2171.

(43) C. R. Cavaglieri, A. Nishiyama, L. C. Fernandes, R. Curi, E. A. Miles and P. C. Calder, Differential effects of short-chain fatty acids on proliferation and production of pro- and anti-inflammatory cytokines by cultured lymphocytes, Life Sci, 2003, 73, 1683-1690.

(44) F. S. Lira, J. C. Rosa, G. D. Pimentel, M. Seelaender, A. R. Damaso, L. M. Oyama and C. O. D. Nascimento, Both adiponectin and interleukin-10 inhibit LPS-induced activation of the NF-kB pathway in 3T3-L1 adipocytes, Cytokine, 2011, 57, 98-106. 
(45) I. Zanoni, R. Ostuni, L. R. Marek, S. Barresi, R. Barbalat, G. M. Barton, F. Granucci and J. C. Kagan, CD14 controls the LPS-induced endocytosis of Toll-like receptor 4, Cell, 2011, 147, 868-880.

(46) K. Meijer, P. de Vos and M. G. Priebe, Butyrate and other short-chain fatty acids as modulators of immunity: what relevance for health?, Curr Opin Clin Nutr Metab Care, 2010, 13, 715-721.

(47) W. Zhao, M. Yuan, P. Li, H. Yan and J. Liu, Short-chain fructo-oligosaccharides enhances intestinal barrier function by attenuating mucosa inflammation and altering colonic microbiota composition of weaning piglets, 2019.

(48) WenXiong, HaoyueMa, ZhuZhang, MeilanJin, JianWang, YuweiXu and ZiliWang, Icariin enhances intestinal barrier function by inhibiting NF-KB signaling pathways and modulating gut microbiota in a piglet model, RSC Advances, 2019, 9.

(49) E. Steed, M. S. Balda and K. Matter, Dynamics and functions of tight junctions, Trends Cell Biol, 2010, 20, $142-149$.

(50) J. Yang, K. Qian, C. Wang and Y. Wu, Roles of Probiotic Lactobacilli Inclusion in Helping Piglets Establish Healthy Intestinal Inter-environment for Pathogen Defense, Probiotics Antimicrob Proteins, 2018, 10, 243-250.

(51) J. Wang, H. Ji, S. Wang, H. Liu, W. Zhang, D. Zhang and Y. Wang, Probiotic Lactobacillus plantarum Promotes Intestinal Barrier Function by Strengthening the Epithelium and Modulating Gut Microbiota, Front Microbiol, 2018, 9, 1953.

(52) E. E. Elamin, A. A. Masclee, J. Dekker, H. J. Pieters and D. M. Jonkers, Short-chain fatty acids activate AMP-activated protein kinase and ameliorate ethanol-induced intestinal barrier dysfunction in Caco-2 cell monolayers, J Nutr, 2013, 143, 1872-1881.

(53) Y. liboshi, Adhesive mucous gel layer and mucus release as intestinal barrier in rats, Jpen Journal of Parenteral \& Enteral Nutrition, 1996, 20.

(54) M. G. Smirnova, L. Guo, J. P. Birchall and J. P. Pearson, LPS up-regulates mucin and cytokine mRNA expression and stimulates mucin and cytokine secretion in goblet cells, Cell Immunol, 2003, 221, 42-49.

\section{Tables}

Table 1. Primer sequences used for Real-time quantitative PCR 


\begin{tabular}{|llll|}
\hline genes & Forward primer (5'-3') & Reverse primer (5'-3') & $\begin{array}{l}\text { accession } \\
\text { number }\end{array}$ \\
\hline MUC1 & GTGCCGCTGCCCACAACCTG & AGCCGGGTACCCCAGACCCA & XM_021089730.1 \\
\hline MUC2 & GGTCATGCTGGAGCTGGACAG & TGCCTCCTCGGGGTCGTCAC & XM_021082584.1 \\
\hline MUC4 & GATGCCCTGGCCACAGAA & TGATTCAAGGTAGCATTCATTTGC & XM_021068274.1 \\
\hline TLR4 & TCAGTTCTCACCTTCCTCCTG & GTTCATTCCTCACCCAGTCTTC & NM_001293316.1 \\
\hline MyD88 & CCCCAGCGATACCCAGTTTGT & ATCCGACGGCACCTCTTTTCA & NM_001099923.1 \\
\hline $\begin{array}{l}\text { NF-KB } \\
\text { p65 }\end{array}$ & AACCCCTTCCAAGTTCCCA & TCCCCGAGTTCCGATTCAC & NM_001114281.1 \\
\hline GAPDH & ATGGTGAAGGTCGGAGTGAAC & ATGGTGAAGGTCGGAGTGAAC & NM_001206359.1 \\
\hline
\end{tabular}

MUC1, Mucin 1; MUC2, Mucin 2; MUC4, Mucin 4; TLR4, Toll-like receptor 4; Myd88, myeloid differentiation primary response 88; NF-KB-p65, nuclear factor kappa B-p65; GAPDH, glyceraldehyde phosphate dehydrogenase.

Table 2. The effect of GOS on the colonic morphology of LPS-challenged suckling piglets

\begin{tabular}{|llllll|}
\hline Items & CON & LPS-CON & LPS-GOS & SEM & $P$-Value \\
\hline Crypt depth & 265.85 & 292.14 & 275.53 & 6.74 & 0.297 \\
\hline Mucosal thickness & 32.86 & 91.62 & 76.30 & 16.49 & 0.529 \\
\hline
\end{tabular}

Values are expressed as mean $\pm S E M, n=6$. CON, control group; LPS-CON, piglets challenged with LPS; LPS-GOS, piglets fed with GOS and challenged with LPS.

Table 3. The effects of GOS on colonic pH value, SCFAs and lactic acid concentrations in colonic digesta of LPS-challenged suckling piglets 


\begin{tabular}{|llllll|}
\hline Items & CON & LPS-CON & LPS-GOS & SEM & $P$-Value \\
\hline $\mathrm{pH}$ value & 6.45 & 6.72 & 6.47 & 0.05 & 0.020 \\
\hline acetate $(\mu \mathrm{mol} / \mathrm{g}$ digesta) & 25.14 & 20.15 & 24.47 & 0.83 & 0.018 \\
\hline propionate $(\mu \mathrm{mol} / \mathrm{g}$ digesta) & 12.60 & 10.06 & 10.99 & 0.56 & 0.181 \\
\hline isobutyrate $(\mu \mathrm{mol} / \mathrm{g}$ digesta) & 1.19 & 1.10 & 1.16 & 0.03 & 0.533 \\
\hline butyrate $(\mu \mathrm{mol} / \mathrm{g}$ digesta) & 6.65 & 4.62 & 6.72 & 0.34 & 0.007 \\
\hline isovalerate $(\mu \mathrm{mol} / \mathrm{g}$ digesta) & 2.10 & 1.90 & 1.97 & 0.12 & 0.804 \\
\hline valerate $(\mu \mathrm{mol} / \mathrm{g}$ digesta) & 2.01 & 1.10 & 1.36 & 0.18 & 0.091 \\
\hline total SCFAs $(\mu \mathrm{mol} / \mathrm{g}$ digesta) & 49.69 & 38.93 & 46.68 & 1.57 & 0.006 \\
\hline lactate $(\mu \mathrm{mol} / \mathrm{g}$ digesta) & 1.58 & 1.26 & 1.62 & 0.06 & 0.021 \\
\hline
\end{tabular}

Values are expressed as mean $\pm S E M, n=6$. CON, control group; LPS-CON, piglets challenged with LPS; LPS-GOS, piglets fed with GOS and challenged with LPS. $P$ values $<0.05$ were considered significant, $P$ values between 0.05 and 0.10 were considered as a tendency. ${ }^{*} P<0.05$, $* \star ~ P<0.01$ compared to the CON group. \# $P<0.05$, \#\# $P<0.01$ compared to the LPS-CON group.

\section{Figures}

a

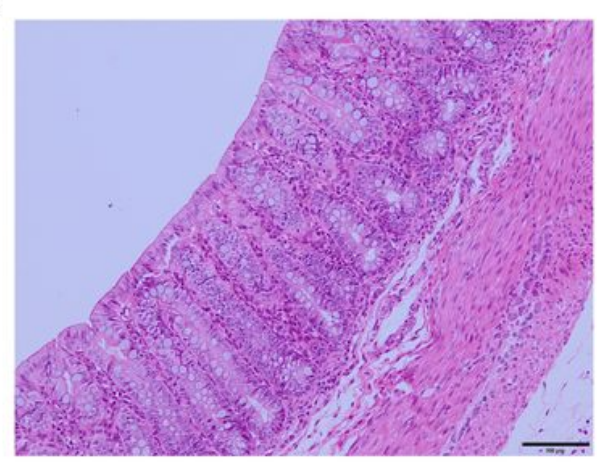

CON b

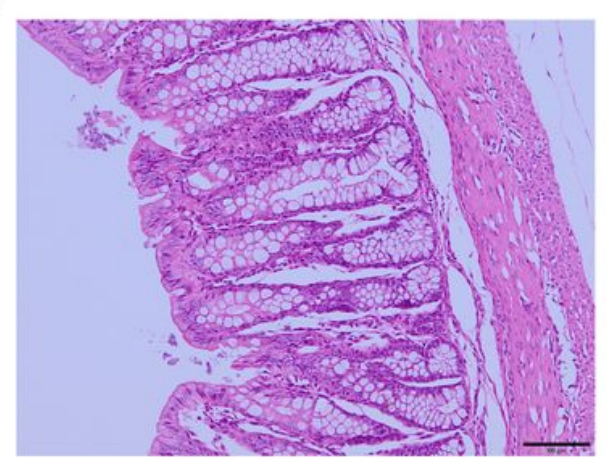

LPS-CON
C

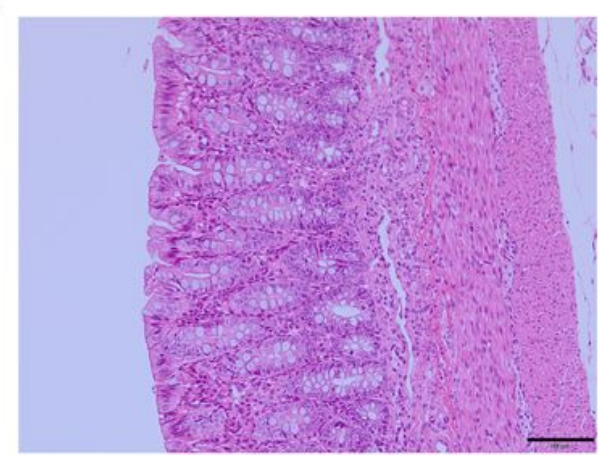

LPS-GOS

\section{Figure 1}

The effect of GOS on colonic epithelial morphology in suckling piglets challenged with LPS. (a) CON, control group. (b) LPS-CON, piglets challenged with LPS. (c) LPS-GOS, piglets fed with GOS and challenged with LPS. 
a

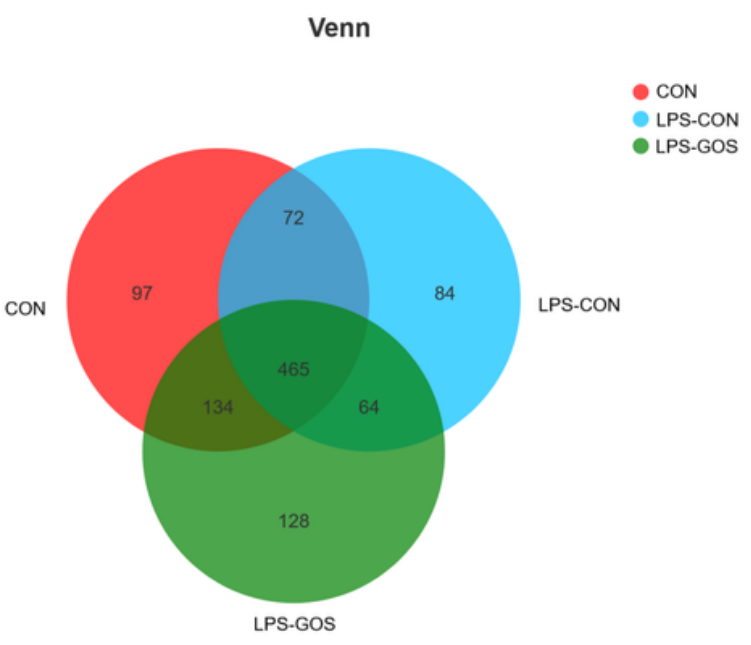

b $\quad$ PCoA on OTU level

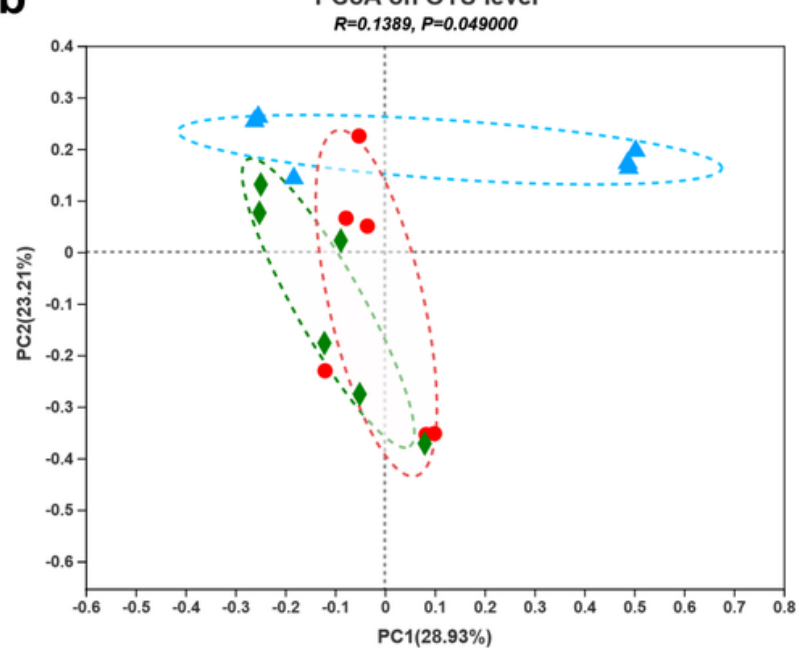

CON

$\triangle$ LPS-CON

LPS-GOS

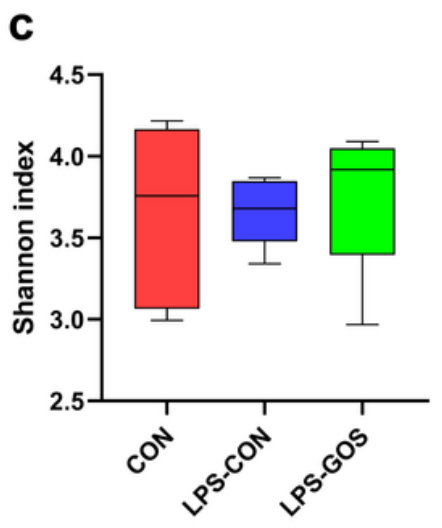

d

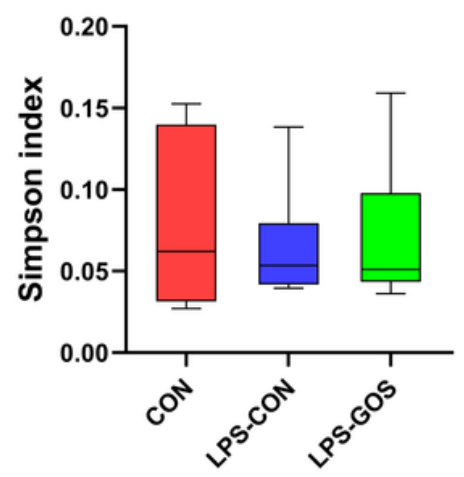

e

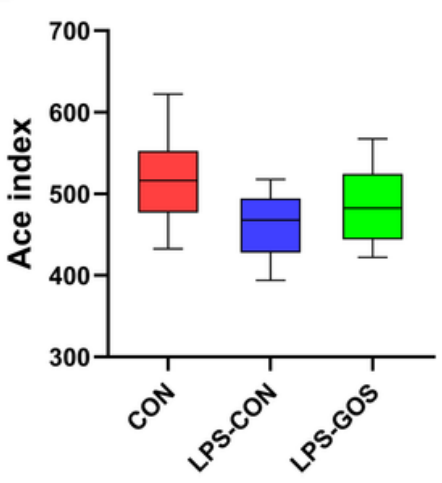

f

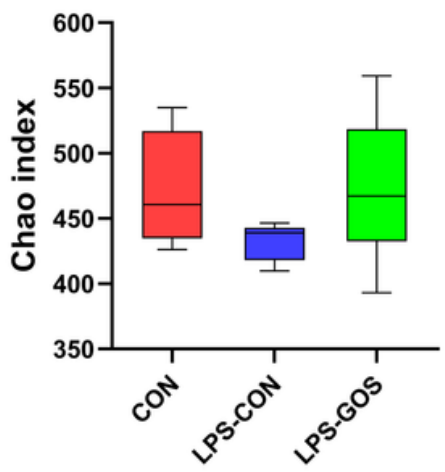

\section{Figure 2}

The effect of GOS on the diversity of colonic mucosa microbiota in suckling piglets challenged with LPS. (a) Venn diagram of core operational taxonomic units in the colonic mucosa. (b) Principal coordinate analysis (PCoA) on colonic microbiota, ANOSIM analysis was used to assess significant differences. (c) the Shannon index of colonic mucosa microbiota. (d) the Simpson index of colonic mucosa microbiota. (e) the Ace index of colonic mucosa microbiota. ( $f$ ) the Chao index of colonic mucosa microbiota. Values are expressed as mean $\pm S E M, n=6$. CON, control group; LPS-CON, piglets challenged with LPS; LPSGOS, piglets fed with GOS and challenged with LPS. 

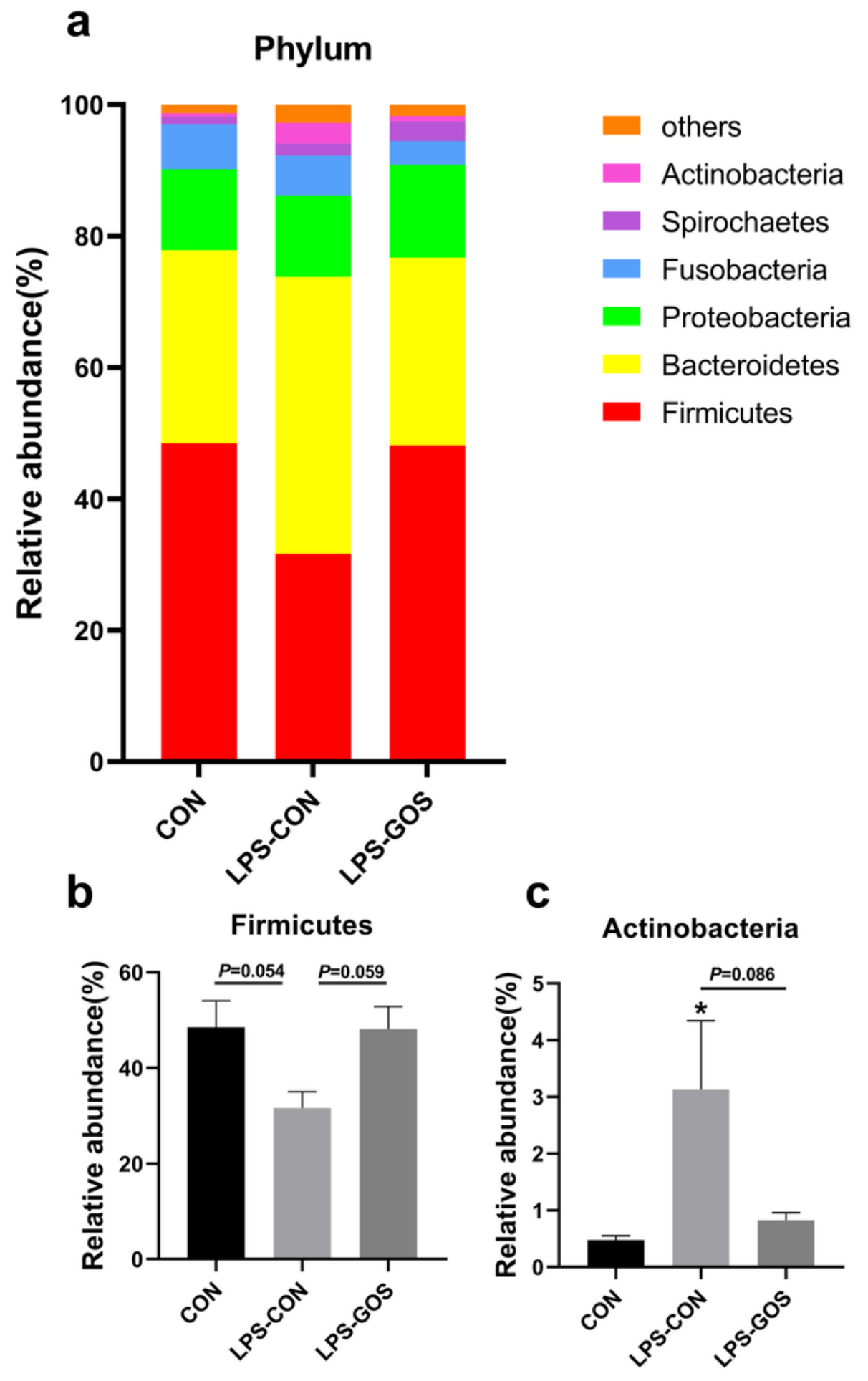

\section{Figure 3}

The effect of GOS on the relative abundance of colonic mucosal microbiota phyla in LPS-challenged suckling piglets. (a) Phylum level composition. (b) The change of Firmicutes. (c) The change of Actinobacteria. $P$ values $<0.05$ were considered significant. Values are expressed as mean $\pm S E M, n=6$. CON, control group; LPS-CON, piglets challenged with LPS; LPS-GOS, piglets fed with GOS and challenged with LPS. * $\mathrm{P}<0.05$ compared to the CON group. 
a
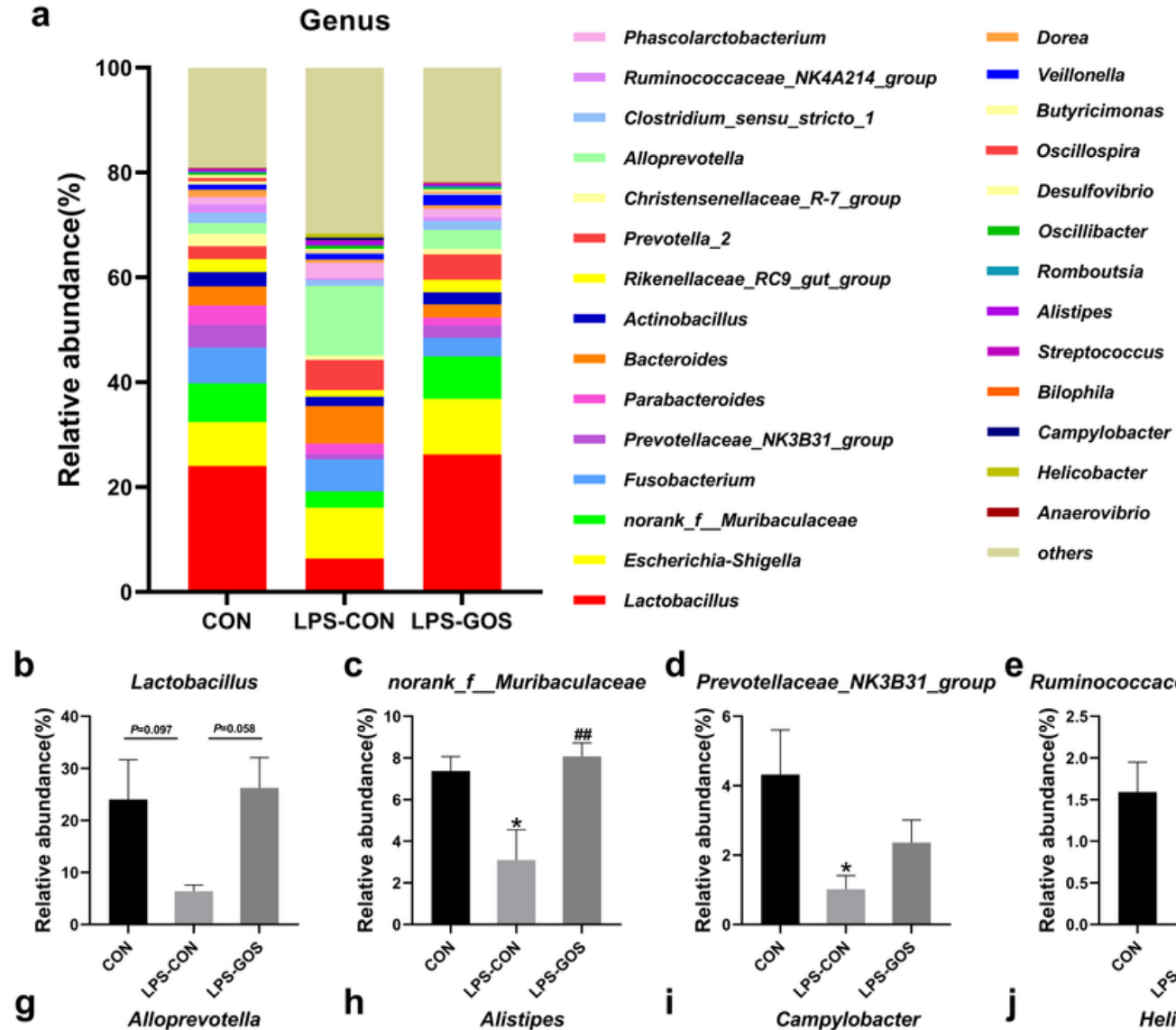

C norank_f_Muribaculaceae

d Prevotellaceae_NK3B31_group

e Ruminococcaceae_NK4A214_group

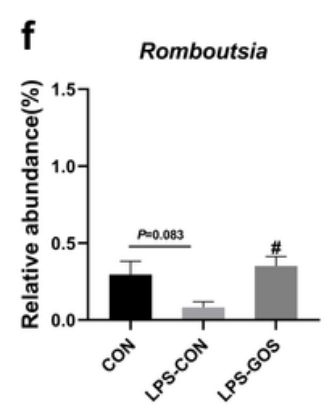

g
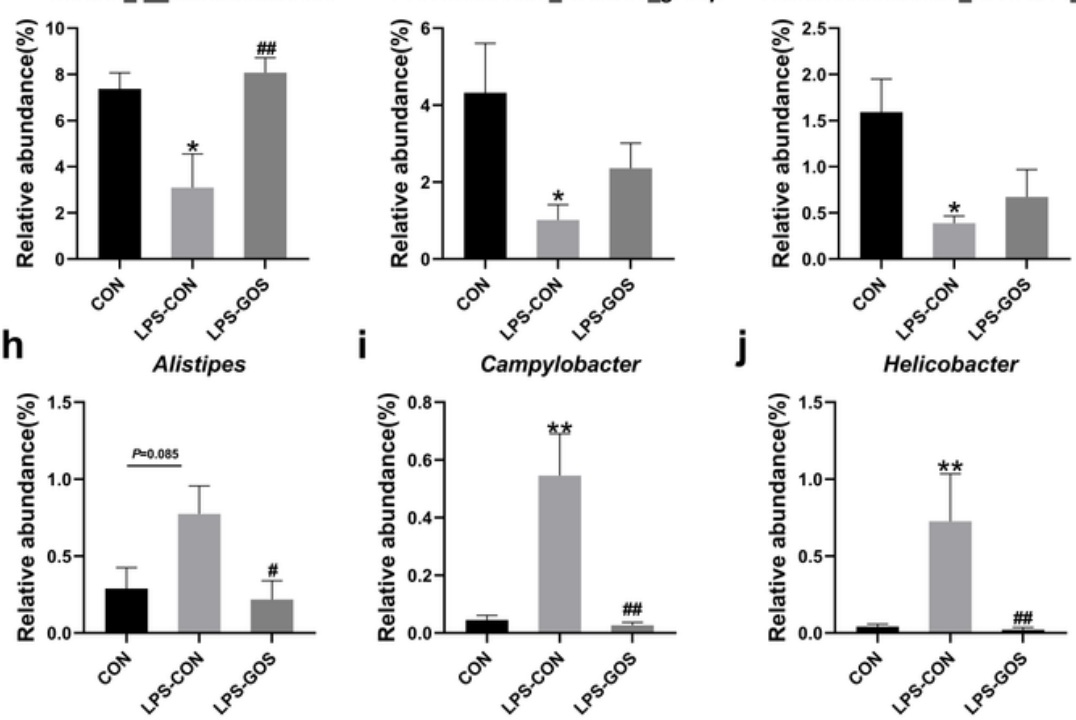

\section{Figure 4}

The effect of GOS on the relative abundance of colonic mucosal microbiota genus in LPS-challenged suckling piglets. (a) Genus level composition. (b) The change of Lactobacillus. (c) The change of norank_f_Muribaculaceae. (d) The change of Prevotellaceae_NK3B31_group. (e) The change of Ruminococcaceae_NK4A214_group. (f) The change of Romboutsia. (g) The change of Alloprevotella. (h) The change of Alistipes. (i) The change of Campylobacter. (j) The change of Helicobacter. P values $<0.05$ were considered significant, $P$ values between 0.05 and 0.10 were considered as a tendency. Values are expressed as mean \pm SEM, $n=6$. CON, control group; LPS-CON, piglets challenged with LPS; LPS-GOS, piglets fed with GOS and challenged with LPS. * $P<0.05$, ** $P<0.01$ compared to the CON group. \# $P<$ 0.05, \#\# P $<0.01$ compared to the LPS-CON group. 
MUC1

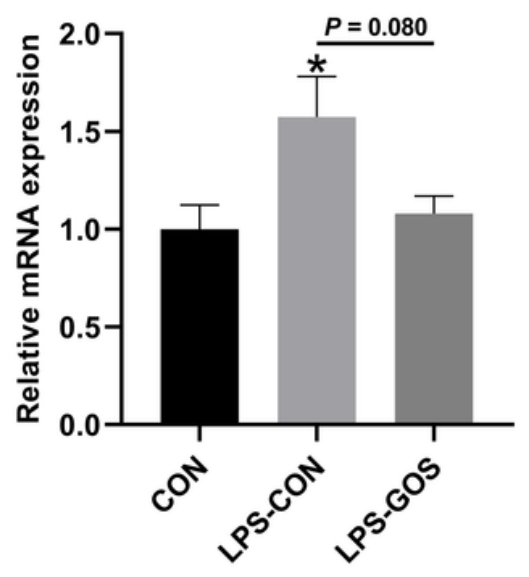

MUC2

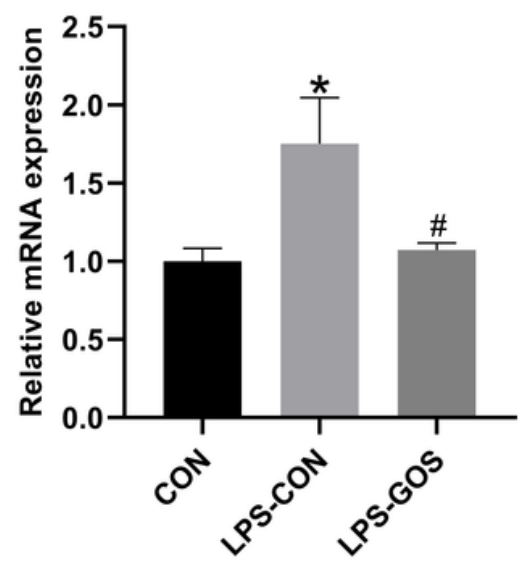

MUC4

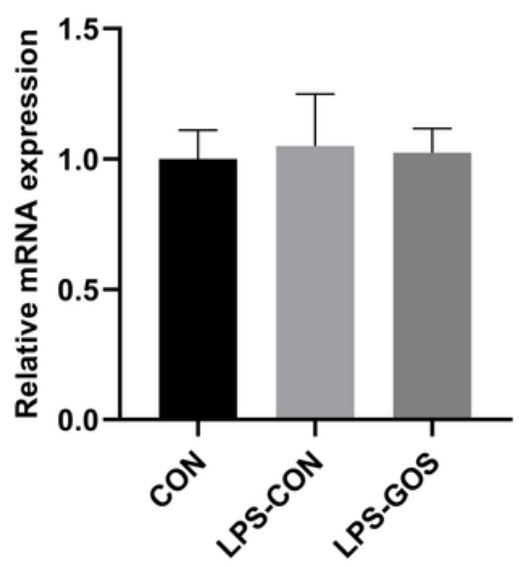

Figure 5

The effect of GOS on the relative mRNA expression of mucin 1 (a), mucin 2 (b) and mucin 4 (c) in the colonic mucosa of LPS-challenged suckling piglets. The values were calculated relative to the expression of GAPDH with formula 2- $\Delta \Delta \mathrm{Ct}$. $\mathrm{P}$ values $<0.05$ were considered significant, $\mathrm{P}$ values between 0.05 and 0.10 were considered as a tendency. Values are expressed as mean $\pm S E M, n=6$. CON, control group; LPS-CON, piglets challenged with LPS; LPS-GOS, piglets fed with GOS and challenged with LPS. * $\mathrm{P}<$ 0.05 , $* * P<0.01$ compared to the CON group. \# $\mathrm{P}<0.05$, \#\# $\mathrm{P}<0.01$ compared to the LPS-CON group.



\section{Figure 6}

The effect of GOS on the relative protein expression of barrier proteins in the colonic mucosa of LPSchallenged suckling piglets. P values $<0.05$ were considered significant. Values are expressed as mean \pm SEM, $n=6$. CON, control group; LPS-CON, piglets challenged with LPS; LPS-GOS, piglets fed with GOS and challenged with LPS. ${ }^{*} \mathrm{P}<0.05$ compared to the CON group. \# $\mathrm{P}<0.05$ compared to the LPS-CON group. 
a
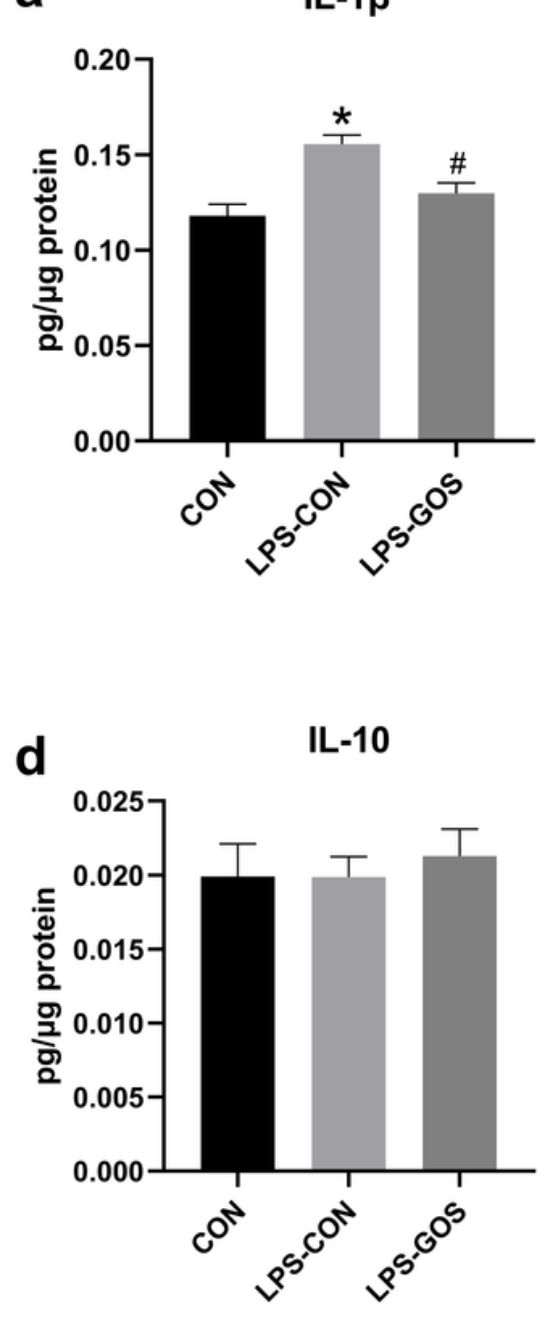

b
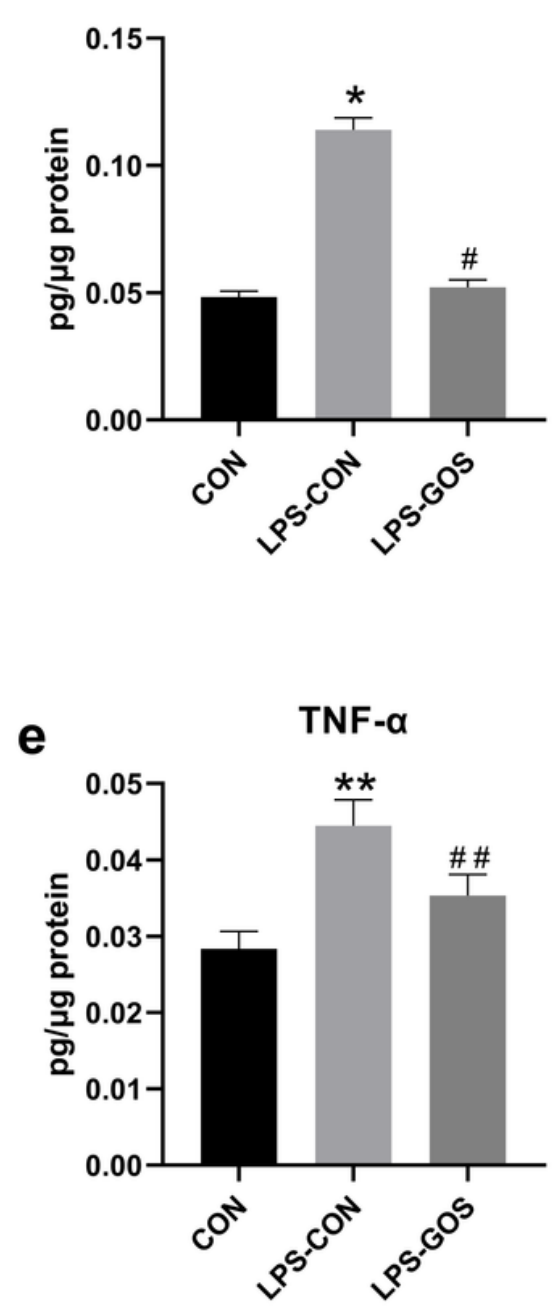

C IL-8

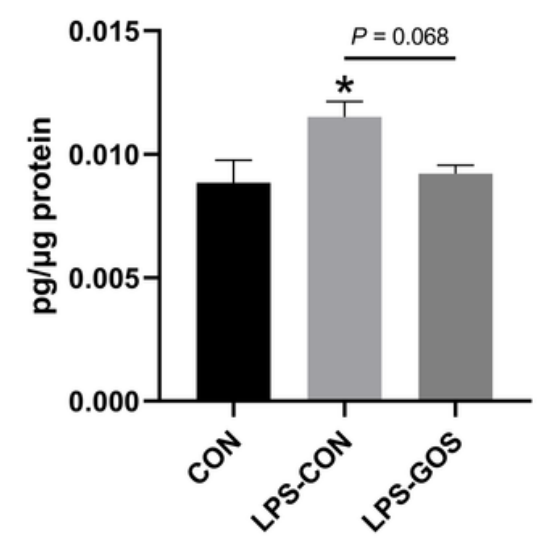

f

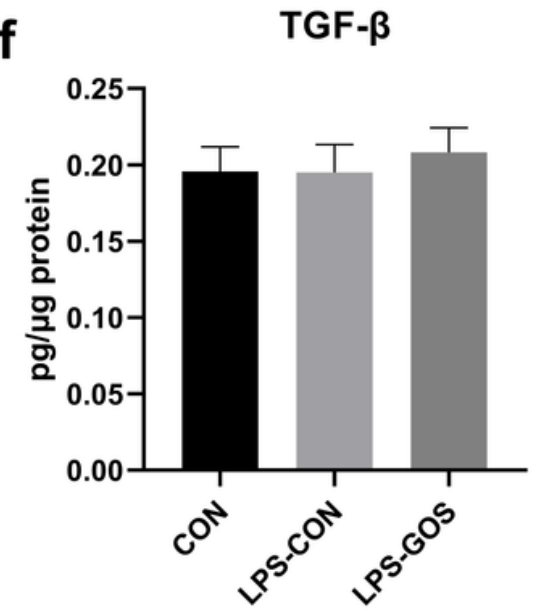

Figure 7

The effect of GOS on the cytokine concentration in the colonic mucosa of LPS-challenged suckling piglets. (a) The change of IL-1 $\beta$. (b) The change of IL-6. (c) The change of IL-8. (d) The change of IL-10. (e) The change of TNF-a. (f) The change of TGF- $\beta$. P values $<0.05$ were considered significant, $P$ values between 0.05 and 0.10 were considered as a tendency. Values are expressed as mean $\pm S E M, n=6$. CON, control group; LPS-CON, piglets challenged with LPS; LPS-GOS, piglets fed with GOS and challenged with LPS. ${ }^{*} \mathrm{P}<0.05$, ${ }^{\star *} \mathrm{P}<0.01$ compared to the CON group. \# $\mathrm{P}<0.05$, \#\# $\mathrm{P}<0.01$ compared to the LPS-CON group. 
a

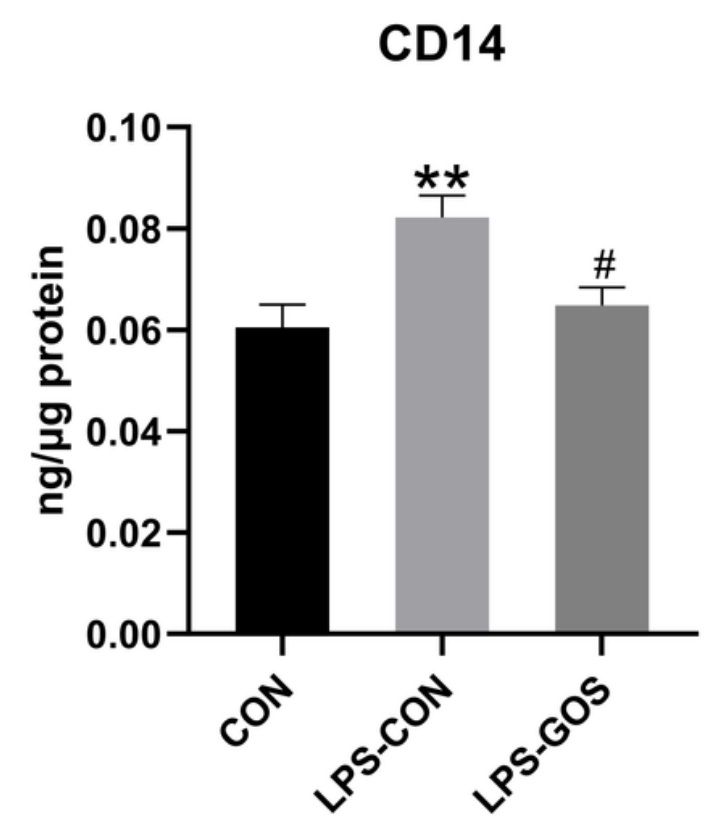

C

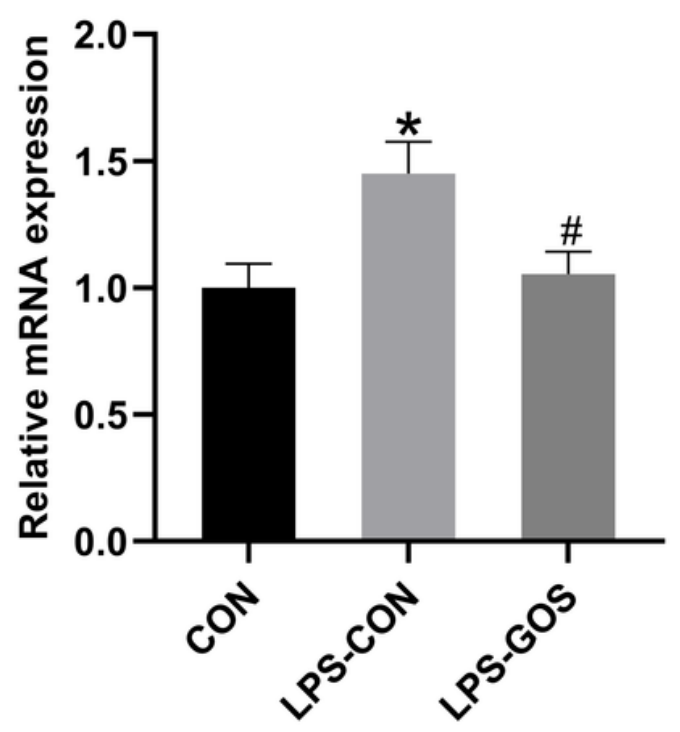

b

TLR4

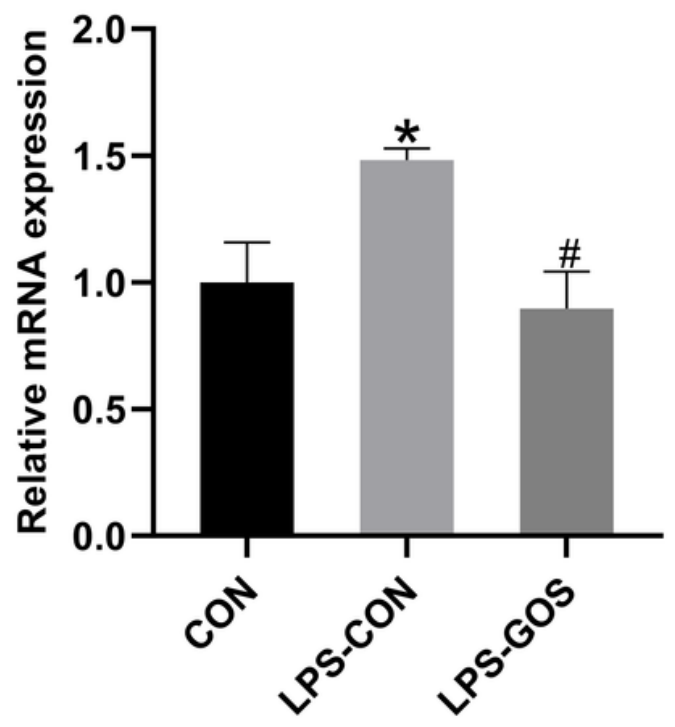

d
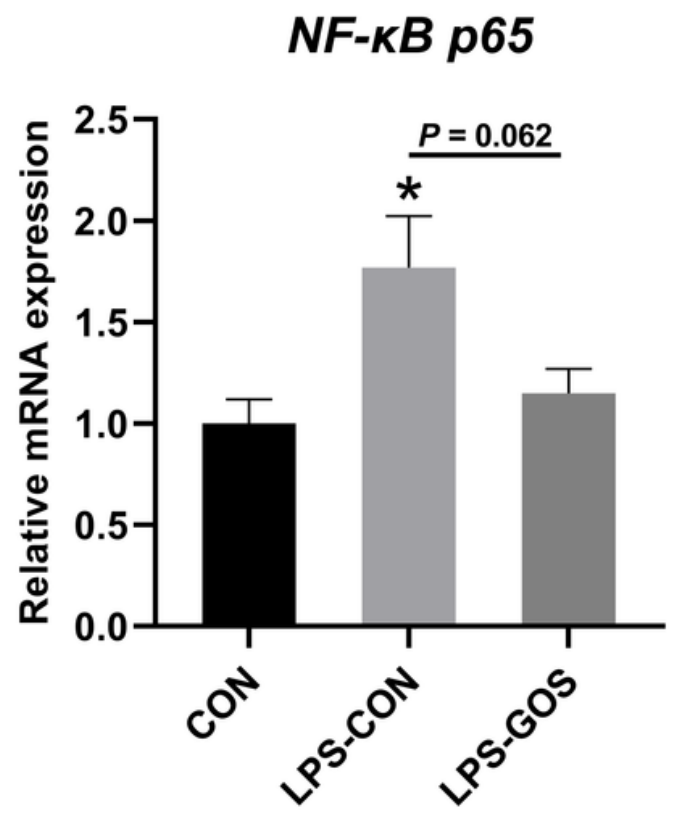

Figure 8

The effect of GOS on the concentration of CD14 (a) and the relative mRNA expression of TLR4 (b), MyD88 (c) and NF-KB p65 (d) in the colonic mucosa of LPS-challenged suckling piglets. P values $<0.05$ were considered significant, $P$ values between 0.05 and 0.10 were considered as a tendency. Values are expressed as mean $\pm S E M, n=6$. CON, control group; LPS-CON, piglets challenged with LPS; LPS-GOS, piglets fed with GOS and challenged with LPS. ${ }^{*} \mathrm{P}<0.05$, $* * \mathrm{P}<0.01$ compared to the CON group. \# $\mathrm{P}<$ 0.05 , \#\# $\mathrm{P}<0.01$ compared to the LPS-CON group. 